\title{
PROBABILISTIC CHOICE: A SIMPLE INVARIANCE
}

J. M. HORNER and J. E. R. STADDON

Department of Psychology, Duke University, Durham, NC 27706, U.S.A.

(Accepted 3 June 1987)

\section{ABSTRACT}

Horner, J. M. and Staddon J. E. R. 1987. Probabilistic choice: A simple invariance. Behav. Processes, 15:59-92

When subjects must choose repeatedly between two or more alternatives, each of which dispenses reward on a probabilistic basis (two-armed bandit), their behavior is guided by the two possible outcomes, reward and nonreward. The simplest stochastic choice rule is that the probability of choosing an alternative increases following a reward and decreases following a nonreward (reward following). We show experimentally and theoretically that animal subjects behave as if the absolute magnitudes of the changes in choice probability caused by reward and nonreward do not depend on the response which produced the reward or nonreward (source independence), and that the effects of reward and nonreward are in constant ratio under fixed conditions (effect-ratio invariance)-properties that $f$ it the definition of satisficing. Our experimental results are either not predicted by, or are inconsistent with, other theories of free-operant choice such as Bush-Mosteller, molar maximization, momentary maximizing, and melioration (matching).

Key words: stochastic model, ratio schedules, pigeon, Bush-Mosteller, reward, satisficing, equilibrium analysis, effect-ratio invariance

\section{INTRODUCTION}

Understanding how reward affects behavior is one of the oldest and most important questions in psychology. A simple way to study this problem is in a symmetrical, two-choice situation with two identical responses each rewarded probabilistically. In such a situation the outcome of each choice-reward or nonreward-is the only information available to the subject, and the objective is to discover the rule by which outcomes affect future choices. We believe that we have discovered a simple property of this rule.

A theory of recurrent choice in probabilistic situations may be quite complicated, involving expectancies and the like, but can nevertheless always be boiled down to two parts: a reward variable to which the organism is presumed sensitive, and a rule or set of rules that links changes in behavior to the value of that variable. In recent treatments of free-operant choice, two kinds of reward variable have been widely discussed: rate and probability. For example, many molar optimality theories assume that animals act so as to maximize reward rate (e.g., Staddon \& Motheral, 1978), and what we may term strong molar maximizing theories (e.g., Rachlin, 1978; Rachlin \& Burkhard, 1978; Rachlin, 
Green, Kagel, \& Battalio, 1976; Rachlin, Kagel, \& Battalio, 1980) assume in addition that animals do this via processes that are directly sensitive to overall reward rate (weak molar maximizing theories are silent on the maximizing process: cf. Staddon \& Hinson, 1983). Other theories, such as the kinetic theory (Myerson \& Miezin, 1980; Staddon, 1977) or melioration (proposed responsible for the matching of response and reward ratios widely observed in free-operant choice experiments: Herrnstein \& Vaughan, 1980; Vaughan, 1981), assume only that animals are sensitive to local (as opposed to overall) reward rate, i.e., rate of reward computed over a time or response window, or over the time period when an animal is actually responding to a particular alternative.

Reward probability has also been proposed as a guiding variable: for example, in the theory of momentary maximizing (Hinson \& Staddon, 1983a, b; Shimp, 1966, 1969; Silberberg, Hamilton, Ziriax, \& Casey, 1978; Staddon, 1980).

Two main kinds of behavior-change rule have been proposed. Melioration assumes that animals will increase the time devoted to the alternative associated with the higher local reward rate, thus tending to equalize the ratio of responses to rewards for all alternatives (matching). Momentary maximizing assumes that animals will always choose the alternative with the higher reward probability.

Stochastic learning models such as those of Bush and Mosteller (1955) and its many derivatives (e.g., Rescorla \& Wagner, 1972; Sternberg, 1963) are different from these aggregative approaches in that they deal with the effects of individual rewards and nonrewards. Stochastic models have not been widely applied to freeoperant data, however (see Vaughan, 1982, and Daly \& Daly, 1982, for recent attempts). The approach we develop in this paper is in this tradition.

There are several reasons to consider an approach to free-operant choice different from current emphases on matching and maximizing. (a) Although matching occurs under many conditions, it does not occur under all (see reviews in Hinson \& Staddon, 1983b; Killeen, 1982; Myers \& Myers, 1977). (b) Deviations from matching are often systematic. For example, Fantino, Squires, Delbrllck, and Peterson (1972), studying pigeons' performance under concurrent variable-interval variableinterval (conc VI VI) schedules, found that response ratios (responses Right/responses Left) are more extreme than scheduled reward ratios (VI value Left/VI value Right) when the VI values are small (high scheduled reward rates) but less extreme when the VI values are large (low scheduled reward rates). Melioration, the mechanism proposed for matching, cannot account for these systematic deviations. (c) Molar maximizing, the idea that animals act so as to maximize reward rate, which is sometimes proposed as an alternative to matching and melioration, is also often violated: on concurrent variable-interval variable-ratio schedules, for example (Herrnstein \& Heyman, 1979); on singlechoice spaced-responding schedules (Staddon, 1965); and on schedules that involve 
a choice between a simple and an adjusting ratio (Lea, 1976).

In exploratory experiments in our laboratory, we have repeatedly turned up results that do not seem explicable by any of the current theories of free operant choice: matching/melioration, molar maximizing, or momentary maximizing. For example, both melioration and momentary maximizing assume that animals are sensitive to local properties (reward probabilities or reward rates associated with each choice). If these variables are the same for both choices, therefore, neither theory makes any point prediction. In several experiments (Horner, 1986) we looked at choice performance under conditions where these local variables were the same for both alternatives, but absolute reward probability varied. Choice performance often appeared to vary systematically as a function of absolute reward probability, which implies a process other than momentary maximizing or melioration .

These experiments used random, probabilistic reward schedules that rewarded each choice response with constant probabilities $p$ (on the Right) and $q$ (on the Left). This procedure is termed a two-armed bandit in the decision-theory literature; it is a variety of concurrent variable-ratio schedule, in the terminology of operant conditioning.

When $p$ and $q$ are equal, both reward probability and reward rate are independent of preference. In one experiment we varied the absolute value of $p$ and looked for systematic changes in the preferences of four pigeons. The animals were exposed to two $p$-values: $p=1 / 75$ or $1 / 20$, in $A B A$ sequence: $1 / 75$ (days $1-3$ ), $1 / 20$ (days 4-8), 1/75 (days 9-10). Sessions were terminated after the 48th food delivery.

Figure 1 shows average preference (proportion of responding on the right choice key: $R /(R+L)$ ) plotted for each animal across days (sessions). During sessions in which $p=1 / 75$, all animals showed a partial preference close to indifference between the two alternatives. But when $p=1 / 20$, all animals shifted across days toward exclusive preference for one or other alternative. As the figure shows, the partial preferences in the $p=1 / 75$ condition were recoverable after the $p=$ $1 / 20$ condition.

When payoff probabilities are the same for both choices, as they were in this experiment, any pattern of preference is consistent with both matching and maximizing accounts: Reward rate is independent of preference (i.e., the proportion of pecks on the right-hand key, which we will call s). Hence any value of $s$ is consistent with molar maximization. Since reward rates for the two choices are just $R(x)=p x$ and $R(y)=p y$, where $x$ and $y$ are the rates of responding to the two keys and $R(x)$ and $R(y)$ are the associated reward rates, matching, $x / y=R(x) / R(y)$, is forced by this procedure. Reward probability is by definition identical for the two choices, so that momentary maximizing (picking the higher-probability-of-reward response) offers no guidance. Thus, neither 


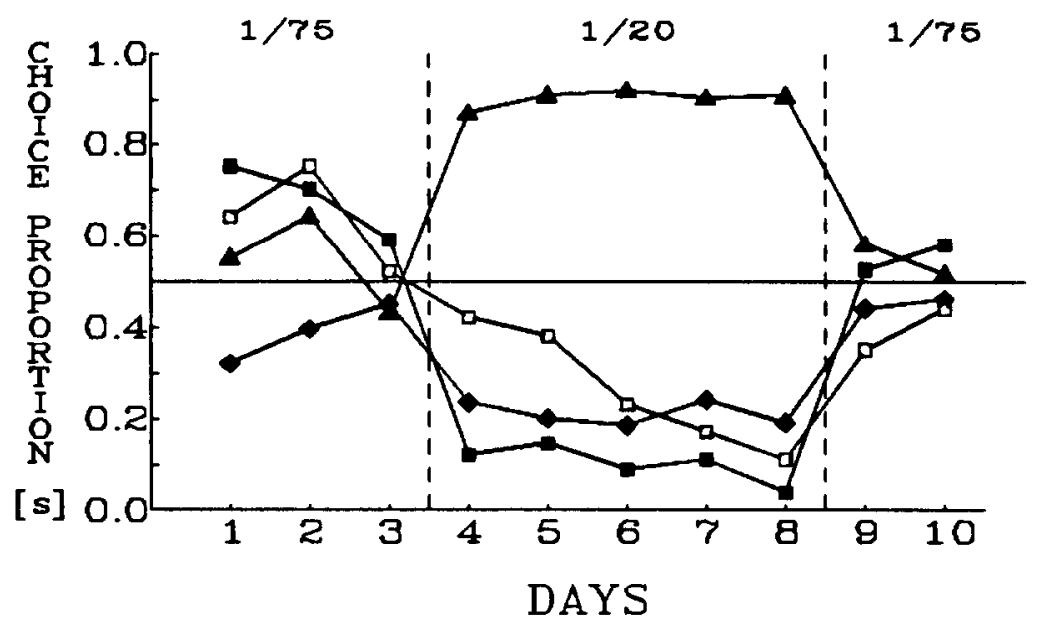

FIGURE 1. The effect of absolute reward probability on choice between identical probabilistic alternatives ("two-armed bandit"). The figure plots the proportion of choices of the right-hand alternative, $s$, across daily sessions for each animal for two conditions, $p=1 / 75$ and $p=1 / 20$, in $A B A$ sequence. Open squares, Bird 096; closed diamonds, Bird 145; closed squares, Bird 151; and closed triangles, Bird 156 .

molar maximizing nor matching (melioration) nor momentary maximizing predicts our result: that low reward probabilities yield indifference between equiprobable alternatives and higher reward probabilities yield exclusive choice.

Unfortunately the pattern shown in Figure 1 is not the only one we have seen with this procedure. In various replications, the exclusive-choice pattern is the commonest, and we have not yet been able to isolate the necessary and sufficient conditions for the simple switch between indifference and exclusivity shown in the figure. In other experiments we have studied choice between probabilistic schedules in which the two probabilities are unequal ( $\neq q)$, and here again we have not infrequently found results contrary to all the standard theories. For example, under some conditions (usually when both probabilities are high), pigeons will sometimes persist for some time in choosing the smaller of the two probabilities. Under other conditions (usually when both probabilities are low) they will sometimes persistently sample both, rather than fixating on the majority side (cf. Allison, 1983). It seems clear from these results that something more complicated than melioration or momentary maximizing is going on. Our reflections on what that might be have led us to a theory that we call ratio-invariant reward following or ratio-invariance, for short. We first describe the theory, show how it can accommodate the full range of results we have obtained with simple probabilistic schedules, and then derive predictions for more sensitive tests with 
an equal-probability (symmetrical) interdependent probabilistic schedule. The first experiment tests these predictions. The second experiment tests predictions for an asymmetrical interdependent schedule.

\section{RATIO-INVAR IANCE}

Our preliminary results with equal-reward-probability alternatives rule out both local reward rate and local reward probability as the variables guiding choice. Accounts such as melioration and momentary maximizing might nevertheless be modified to fit these data by adding explicit dynamic assumptions about averaging windows and the like. There is a simpler alternative, however, derived from the most primitive construction of the law of effect, namely the assumption that a rewarded choice increases in probability, while an unrewarded choice decreases in probability.

To show how a particular version of this idea is consistent with our initial results it needs to be made more explicit. We derive predictions intuitively here; a more formal development is given in Appendix A. We begin with the standard assumption of stochastic learning models: that each reward increments s, the probability of the rewarded response, and each nonreward (unrewarded response) decrements this probability. A useful form of this assumption is that the increment owing to reward is an amount a(s) that will in general be some function of $s$, and that the decrement owing to each nonreward is an amount $b(s)$.

We can define a quantity delta(s), the expected change in choice proportion, $s$, associated with the four possible choice-reward combinations. Consider a set of responses, allocated in the proportion $s$ and $1-s$ to Right and Left keys, respectively, and let us look at the relative contribution to delta(s) of the four possible outcomes of responding: reward and nonreward for responses to Right and Left. Let the reward probabilities on Right and Left be $p$ and $q$, respectively. Then the contributions to delta(s) of the four possible outcomes are as follows:

$$
\begin{array}{r}
\text { Reward on R: } \quad a(s) p s \\
\text { Reward on L: }-a(s) q(1-s) \\
\text { Nonreward on R: }-b(s)(1-p) s \\
\text { Nonreward on L: } \quad b(s)(1-q)(1-s),
\end{array}
$$

where delta(s) is just the sum of terms la-d. Note that $s$, the proportion of $R$ (right) choices, is incremented by both reward on $R$ and nonreward on $L$ and decremented by the other two possibilities. In words, Eq. la says that the expected change in $s$ associated with a rewarded $R$ response is equal to the probability of such a co-occurrence, ps, multiplied by the change associated with a rewarded response, $a(s)$, and similarly for the other equations. Suming these 
four terms yields:

$$
\operatorname{de} 1 \mathrm{ta}(s)=a(s) p s-a(s) q(1-s)-b(s)(1-p) s+b(s)(1-q)(1-s),(2 a)
$$

which yields after rearrangement the following expression for delta(s) as a function of $s, p, q, a(s)$ and $b(s)$ :

$$
\operatorname{delta}(s)=s[(p+q)(a(s)+b(s))-2 b(s)]+b(s)-q[a(s)+b(s)] .(2 b)
$$

Another way to think of Eq. $2 \mathrm{a}$ is in terms of the absolute number of rewards and nonrewards on each side. If the number of rewards on Left and Right is denoted by $R_{R}$ and $R_{L}$, and the number of nonrewards by $N_{R}$ and $N_{L}$, then Eq. 2a can also be written

$$
\operatorname{delta}(s)=\left\{a(s)\left[R_{R}-R_{L}\right]-b(s)\left[N_{R}-N_{L}\right]\right\} / s,
$$

where $S$ is the total number of responses to both sides. Equation $2 \mathrm{c}$ shows the symmetry of the model, and makes it obvious that delta(s) is zero (a potential equilibrium) only when the difference between the (weighted) differences in number of rewards and nonrewards on both sides is equal.

Equation 2 represents a general class of novel reward-following models, defined by the property that the absolute value of the change in choice probability following a response depends only on the outcome event, reward or nonreward, and not on its source (a Left or Right choicc). At a given s-value, reward for a Right response increments $s$ by the same amount that a reward on the Left decrements it--and similarly for a nonreward. The source of the outcome affects on ly the sign of the change. $L t$ is as if the subject generates $R$ and $L$ choices via a stochastic process that has a single probability setting, whose value is then altered up or down by the quantities $a(s)$ and $b(s)$, depending on whether the outcome is reward or nonreward on $L$ or $R$.

This property, which might be termed source-independence, is violated by the standard linear operator models, since the effects of reward and nonreward are there assumed to act differently on s depending on which choice was made: In the Bush-Mosteller model, for example, reward on the right increments $s$ by an amount $A(1-8)$, whereas reward on the left decrements $s$ by an amount As (where $A$ is a constant, $0<A<1$ ), which will be the same on ly when $s=1 / 2$.

So far we have said nothing about the details of $a(s)$ and $D(s)$, the changes in choice probability associated with reward and nonreward. (It might seem necessary that $a(s)$ and $b(s)$ be constrained in such a way that $s$ remain within the interval 0 - 1 , but this is not in fact essential to the derivation of meaningful equilibrium predictions.) It turns out that we need not know the form of a(s) and 
$b(s)$ in order to derive equilibrium predictions. Let us consider how a(s) might depend on s. Bush-Mosteller assumes that a(s) will differ depending on whether reward is for a left or a right response. For example, if $s$ is close to zero, reward for a right response has a large effect, reward for a left response a negligible effect, with the opposite being true for nonreward. The absolute magnitude, as well as the sign, of the effect of reward or nonreward on preference thus depends on the source of the event. Moreover, reward and nonreward vary reciprocally: When reward has a large effect, nonreward has a small one and vice versa. These asymmetries do not seem plausible. It may be at least as reasonable to assume that the subject has a fixed degree of certainty about the properties of each alternative in a symmetrical situation: If he is relatively certain, then reward and nonreward, for either choice, will both have a small effect; conversely, if he is uncertain, both will have a large effect. In other words, it seems reasonable to consider the possibility that the ability of both reward and nonreward to affect preference depends in a similar way on the value of $\mathrm{s}$.

The simplest possible assumption consistent with the intuition of similar dependence on $s$ is just that the ratio $a(s) / b(s)$ is approximately constant for all s-values. It is easy to show that this assumption is by itself sufficient to generate equilibrium predictions from Eq. 2, even without any knowledge whatever about the form of $a(s)$ (and $b(s)$ ). The proof is as follows. If $a(s) / b(s)=v, a$ constant, then so is the quantity $w=b(s) /[a(s)+b(s)]=1 /(v+1)$, which we term the effect ratio. Dividing both sides of Eq. 2 by the quantity $[a(s)+b(s)]$ and making the substitution yields:

$$
[\operatorname{delta}(s)] /[(a(s)+b(s)]=s(p+q-2 w)+w-q \cdot
$$

If we are interested only in the equilibrium properties of this process, we need to know only the s-values for which delta(s) is zero and the slope of the delta(s) function at those points. (In the absence of more information about $a(s)$ and $b(s)$, we must forego predictions about the shape of the acquisition function, the number of responses needed to achieve equilibrium, or the variance of the choice distributions.) For an equilibrium analysis, the positive multiplier $1 /[a(s)+b(s)]$ on the left-hand side of the equation can therefore be ignored, which yields the following fundamental relation:

$$
\operatorname{delta}(s)=s(p+q-2 w)+w-q .
$$

The comparable derivation from Eq. $2 c$ yields

$$
\operatorname{delta}(s)=v\left[R_{R}-R_{L}\right]-\left[N_{R}-N_{L}\right]
$$


We term the process from which Eq. 3 is derived (constancy of the ratio of the effects of reward and nonreward) effect-ratio-invariant reward following, or ratio invariance, for short.

\section{Deriving predictions}

The simplest way to derive the equilibrium predictions of ratio invariance is to plot delta(s) (i.e., Eq. 3a, which for the simple two-armed bandit is linear in $s$, but for more complex procedurcs has other forms, as we show in a moment) across all values of choice proportion, s, for fixed values of $a, b, p$, and $q$. At the point where this function crosses the abscissa, delta(s) is equal to zero; this point is therefore a potential equilibrium.

Figure 2 shows a plot of this type for the first condition in our pilot experiment (i.e., $p=q=1 / 75$ ); we set $w$, the effect ratio, at 0.03 , a value in the range we have established from other experiments. Under these conditions, i.e., when $p=q$, Eq. 3 crosses the abscissa at the point $s=0.5$ : indifference. Indifference represents a stable equilibrium here because the line has a negative slope (i.e., the quantity $(p+q-2 w)$ is negative because $p, q<w$ ), so that small perturbations of $s$ in either direction from the point $s=0.5$ produce delta(s) values that oppose the change.

Figure 2 also shows the prediction for the other condition in the pilot experiment, where $\mathrm{p}=\mathrm{q}=1 / 20$ (which is greater than our w-value of 0.03 ). Here the equilibrium solution is at $s=0.5$, as before, but the equilibrium is unstable, because the slope of the line is positive (perturbations of $s$ in either direction are amplified, not opposed by the resulting change in $s$ ). Consequent $1 y$, the predicted outcome is exclusive choice, at $s=0$ or $s=1$.

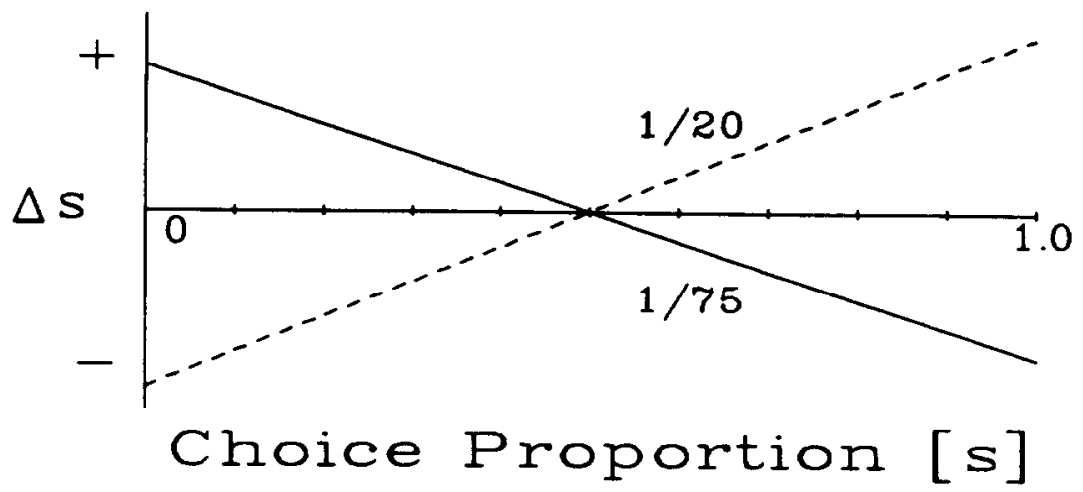

FIGURE 2. Prediction of ratio-invariance for two equal probabilistic schedules. The figure plots delta(s)--calculated from Eq. 3--as a function of the choice proportion, $s$, for $p=1 / 75$ (solid line) and $p=1 / 20$ (dashed line). The effect ratio (see text), w, is 0.03 . 
Thus, the major results from the pilot experiment--exclusive choice when $p$ is high, indifference when $p$ is low--are consistent with ratio invariance. ${ }^{1}$ Neither matching, nor momentary maximizing, nor molar maximization can predict these results, because on the equal-probability schedule any pattern of choice is consistent with all theories. Bush-Mosteller, and its derivatives, cannot predict these results either (it predicts stable indifference for all p-values, as shown in Appendix A).

of course, we have not always obtained the simple switching pattern, but this is not clear disproof of ratio invariance because the prediction of switching depends upon relative constancy of the effect ratio, w, between conditions (see following discussion). And in any case, the switching effect is so simple that it is probably consistent with a great many simple processes. For these reasons, the pilot experiment is a poor test of the theory. More powerful tests of ratioinvariance require procedures in which competing theories make specific, different predictions for the same situation (i.e., under conditions where we can assume constant $w$ ). A procedure in which the results predicted depend in some clearly defined way on $w$ would also be desirable. Experiments 1 and 2 used such procedures.

EXPERIMENT 1 - Preference Patterns on a Symmetrical Interdependent Schedule

The quantity $w$, which represents the relative effect of nonreward and reward, $b(s) /[a(s)+b(s)]$, is obviously critical to the experimental predictions of our analysis. There is reason to believe that $w$ will differ from individual to individual. (or even in the same individual under different conditions). For example, food is not rewarding for a satiated animal. Food and no food are all the same to it--it acts as if $w$ is close to $0.5--$ whereas for a very hungry animal w will obviously be small, because the effects of reward will be large relative to the effects of nonreward. Anything that affects the animal's motivational state is likely to affect the value of w. Since we cannot equate exact ly the motivational states of our animals, w is certain to differ among animals. Consequently, any experimental test of our analysis must be such as to allow for

1. There is obviously a third possibility, namely, $p=w--$ for which ratio invariance makes no point prediction (i.e., there is no unique equilibrium svalue). Our experience leads us to think that this condition may be hard to achieve in practice, however, because $w$ is not independent of such things as overall reward rate and the animal's motivational condition. When the animal can directly affect reward rate (as on the probabilistic schedules studied here) the $p$ $=w$ condition may therefore be an unstable one. We should also note that our analysis can predict the mode(s) of the steady-state choice distribution, but not its variance, which will depend upon the unknown functions $a(s)$ and $b(s)$. For the equal-probability two-arm-bandit situation, the prediction is therefore for a mode either at $s=0.5$ or $s=1$ or 0 , depending upon whether $w$ is greater or less than p. 
individual variation in $w$. It is also quite possible that $w$ can vary as a function of the animal's history. Thus, group-average data and between-conditions comparisons are ruled out. We need procedures that allow us to predict patterns of individual behavior in a fixed situation.

A slight generalization of Eq. 3 suggests a fruitful approach. If, instead of being constant, we allow the reward probabilities $p$ and $q$ to be functions of the actual choice proportion, $s$ (we define how $s$ is measured in a moment), then Eq. 3 becomes

$$
\operatorname{delta}(s)=s[p(s)+q(s)-2 w]+w-q(s)
$$

Once $p(s)$ and $q(s)$ are defined, we can use Eq. 4 as before to find stable and unstable equilibria. In other words, we can generate a class of new procedures for each of which Eq. 4 now makes equilibrium predictions. These procedures are termed interdependent schedules, because the reward conditions for each response depend upon the frequency of that response relative to other responses. They formally resemble the $N$-person game known as the multiperson prisoner's dilemma (Schelling, 1978), or frequency-dependent selection in population biology.

By choosing $\mathrm{p}(\mathrm{s})$ and $\mathrm{q}(\mathrm{s})$ properly, we can pit ratio invariance against any desired alternative theory. It turns out that reward schedules appropriate for this purpose are often predicted to have equilibrium points that are highly constrained, so that these schedules provide powerful tests of ratio invariance, as well as making it possible to discriminate between ratio invariance and other choice theories.

If reward probability is to be a function of choice proportion, $s$, the controlling apparatus for these interdependent schedules must obviously measure s over some number, $M$, of choices preceding the current choice. For Experiments 1 and $2, M$ was 32 , but there seems to be nothing special about that value. In other experiments we have used M-values from 4 to 96 , with essentially similar results. The main effect of $M$ seems to be on the variance of the s-value computed by the schedule, which affects the relative magnitudes of the predicted preference modes, but not their location (see General Discussion). We will report elsewhere parametric data on the effect of $M$.

In this experiment we set $p(s)=q(s)$, i.e., the reward probabilities were always the same for both responses (symmetrical interdependent--SI--schedule). The inverted- $V$ in the top panel of Figure 3, which is a plot of $p(s)$ against $s$, shows the triangular (bilinear) reward function we used in Experiment 1, which consists of two linear segments with a maximum payoff probability at indifference (i.e., $s=0.5: p(.5)=0.085$ ) and two minima at exclusive responding (i.e., $s=$ 0 and $s=1: p(0)=0.005$ and $p(1)=0.045)$. In other words, exclusive choice of the left key yields very low payoff probabilities on both keys, whereas exclusive 




Choice Proportion [s]

FIGURE 3. Top panel: Reward function for the symmetrical interdependent schedule. The figure plots the probability of reward on both alternatives, $p(s)$, as a function of the proportion of choices of the right-hand alternative, $s$ (s was computed over over the preceding 32 choices in this experiment.) Bottom panel: Prediction of ratio-invariance for the symmetrical interdependent schedule for three w-values. The figure plots delta(s) as a function of $s$, calculated from Eq. 4, by substituting the reward function shown in the top panel for $p(s)$ and $q(s)$ (Eq. B3). The three curves were generated with w values of .085 (curve I), 0.58 (curve II) and 0.32 (curve III). Stable equilibria for curve III are at points $d$ and $e$, for curve II at points $b$ and $c$, and for curve $I$ at point a (indifference).

choice of the right key yields an intermediate payoff probability on both; payoff is maximal when the animal chooses each key equally often, i.e., when in every block of 32 choices 16 are of the left and 16 of the right key.

When reward probability is always the same for both choices, matching, momentary maximizing, and (unconstrained) molar maximizing, and ratio-invariance make different predictions. Equal reward probability on both sides means that the animals must always match (the procedure forces $R(x) / x=R(y) / y$, where $x$ and $y$ are response rates on the two choices and $R(x)$ and $R(y)$ are the reward rates obtained, all computed over the same time denominator: hence $x / y=R(x) / R(y)$-matching). 
Hence, matching makes no prediction about preference--other than that it should be variable, perhaps. Similarly, since both alternatives always yield the same payoff probability, simple momentary maximizing makes no prediction. Molar maximization predicts that animals should tend towards indifference $(s=0.5)$, however, because that is where reward probability is a maximum with the bilinear reward function we used. (We chose a bilinear schedule with maximum at $s=0.5$ because, with equal payoff probabilities for both choices, the maximizing solution is also the intuitively most plausible solution. We also recognize that with the appropriate choice of constraints--on memory, for example--molar maximizing might well predict outcomes other than simple indifference. We discuss only unconstrained maximization here.)

Predictions for ratio-invariance can be derived by substituting $p$ (s) (i.e., the bilinear function in Figure 3) for $p(s)$ and $q(s)$ in Eq. 4. A plot of the resulting delta(s) functions for three $w$ values is in the bottom panel of Figure 3. As in Figure 2, we look for zeroes and the function slope at those points to identify stable and unstable equilibria. For curve II ( $w=0.058)$, delta(s) is zero at indifference (i.e., $s=0.5)$, and also at the two points $b(s=0.36)$ and $c(s=0.78)$. The slope of the curve is positive at indifference (so long as $p(.5)>w$ ), indicating an unstable equilibrium, but negative at points $b$ and $c$ indicating that these two points are stable equilibria. It is also possible to show analytically that these two s-values are associated with the same probability of reward, $p(s)$. (See Appendix $B$ for formal derivations.)

The top panel of Figure 3 summarizes the equilibrium predictions of ratio invariance for the bilinear SI schedule for all w values. The horizontal line labelled $w_{1}$ shows the prediction when $p(s)$ is everywhere less than $w$, namely, indifference (point $A$ in the figure: the analysis is essentially the same as in Experiment 1). the horizontal line labelled $w_{2}$ shows the prediction when $w$ is within the range of permitted $p(s)$ values on both sides of the maximuin: stable equilibria at the points labelled $\mathrm{B}$ and $\mathrm{C}$, which are connected by broken lines to the corresponding zeroes of the solid delta(s) function in the bottom pane 1 (points $b$ and $c$ ). $B$ and $C$ are flanked by inward-pointing arrowheads to indicate that they are attractors (strictly speaking, limit points). Point A, the indifference point, is an unstable point. The third horizontal line, labelled $w_{3}$, shows the prediction when $w$ is within the range of $p(s)$ values on one side of the delta(s) function, but below the permitted $p(s)$ values on the other side. As before, point $\mathrm{D}$, where $\mathrm{p}(\mathrm{s})=\mathrm{w}$, is an attractor, so a choice mode is predicted at that point. But there is no $p(s)$ value equal to $w$ on the right-hand side of the delta(s) function, which is everywhere greater than $w$. Since the indifference point is always unstable, a choice mode is predicted here at exclusive choice, $s=$ 1 (point E); delta(s) functions corresponding to all three w-values are shown in 
the bottom panel.

In summary, there are only three choice patterns on the SI procedure that are compatible with ratio invariance: indifference, bimodal choice with each mode at the same $\mathrm{p}(\mathrm{s})$ value (on opposite sides of the maximum), or bimodal choice with one mode at a low s value corresponding to $p(s)$ below the minimal $p(s)$ on the other side of the maximum, and the other mode at exclusive choice ( $s=1$ for the positive-slope condition).

METHOD

Subjects

Four adult White Carneau pigeons served. Each bird was maintained at $85 \%$ of its free-feeding weight, and all had previous experience with various schedules of reinforcement.

\section{Apparatus}

Al1 experiments were performed in a ventilated, sound-attenuating, aluminum and Plexiglas operant-conditioning chamber measuring $30 \mathrm{~cm}$ long $x 28 \mathrm{~cm}$ wide $x 33 \mathrm{~cm}$ high. Three translucent keys, $2 \mathrm{~cm}$ in diameter, were fixed in a triangular arrangement, apex upward, on one wall. The keys were $5.5 \mathrm{~cm}$ from each other, the two lower keys were $20.5 \mathrm{~cm}$ from the floor, and the top key was located $25.5 \mathrm{~cm}$ from the floor. Each key was transilluminated by a 28-w white light. Directly below and on the same wall as the three keys was a $4 \times 5-\mathrm{cm}$ aperture for a food magazine. The food aperture was $8 \mathrm{~cm}$ below the two lower keys. Reward was 2-sec access to grain. During the reward operation all keylights were turned of $f$ and a 28-w light above the hopper was turned on. The experiment was controlled by a SYM microcomputer that recorded individual experimental events and their times to within 1/50th of a second. Data from each experimental session were transferred to another computer for analysis.

\section{Procedure}

We used a choice procedure intended to equate the effortfulness of "staying" (two successive responses on the same key) and "switching" (two successive responses on different keys). Each choice involved two key-pecks. The first peck was on the lighted top key, a single peck on the top key turned it off and turned on the two bottom keys. A peck to either of the two bottom keys turned both off, delivered a reward if one was scheduled, and turned on the top key again. This two-response procedure was used in all experiments, but we varied between experiments the rule that determined the probability of reward for pecks on the two bottom keys.

In Experiment 1, the probability of reward, $p(s)$, was always the same for both choices; $p(s)$ was determined by the proportion of choices, $s$, over the preceding 32 choices according to the bilinear reward function shown in Figure 3 . The controlling computer calculated s after every choice and determined the probability of reward for that choice accordingly.

The first 50 responses at the beginning of each session were not rewarded; rewards were delivered according to the symmetrical interdependent schedule thereafter. Sessions terminated after the $70 \mathrm{th}$ reward.

The experiment ran for 10 daily sessions. 
RESULTS

Figure 4 shows details of the kind of behavior typical of the interdependent schedule. The four rows show comprehensive data for four successive days for a single animal. The left panel in each row summarizes the entire experimental session in the form of a preference trajectory: successive L-choices are represented along the abscissa, successive $R$-choices along the ordinate. Rewards for $L$ and $R$ choices are shown as blips below and above the line, respectively. The center panel in each row shows the value of $s$ (i.e., $R /(L+R)$ over the preceding 32 choices) choice-by-choice throughout the session. The right panel in each row shows the distribution of $s$ averaged across the whole session; superimposed on this distribution is the bilinear reward function used in this experiment .

Figure 4 shows three patterns typical of this procedure: (a) On the first day (top row) the animal shows a stable preference for the left alternative: the trajectory has a slope that varies little throughout the session, and the choice distribution shows a single mode. (b) on the second and third days (rows two and three) the choice trajectory shows a variable slope, the s-value (bottom right) varies systematically through the session and the choice distribution (bottom left) is multimodal. (c) On the fourth day (bottom row) the animal again shows a stable preference (this time for the right choice).

Figure 4 suggests that there are two stable preference modes for bird 096 on this interdependent schedule. This impression is confirmed in Figure 5, which shows the percentage of total number of responses at a particular proportion of choices (computed in a running "window" of 32 responses) for each animal (i.e., the panels show the distribution of choice proportion, $s$, as defined by the SI procedure). These graphs are made by collapsing across sessions for the entirety of the experiment. Although each animal showed different degrees of preference for the two alternatives, all animals were similar in two respects. First, all preference distributions were bimodal. Second, none of the animals had a modal preference for indifference, where there was the highest probability of reward.

Table 1 gives the choice proportion, $s$, and the probability of reward, $p(s)$, for each animal's modal preference. Note that the bimodal preference distributions are not symmetrical; the modal s-values for each animal tend to fall at the same probability of reward, $p(s)$. Because the reward function is not perfectly symmetrical, such points are not at equal distances from the 0.5 point. The major exception to this tendency for both modes to be at the same $p(s)$ value is animal 156 . 

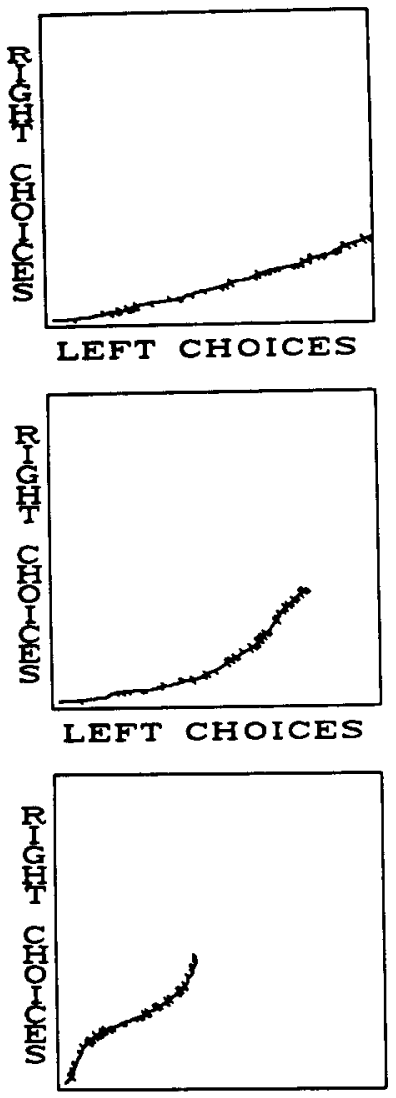

LEFT CHOICES

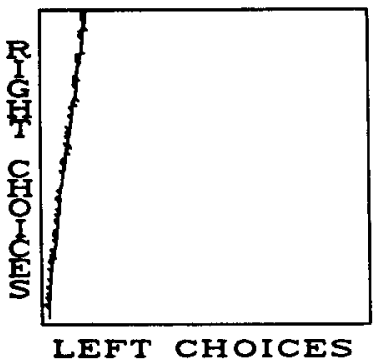

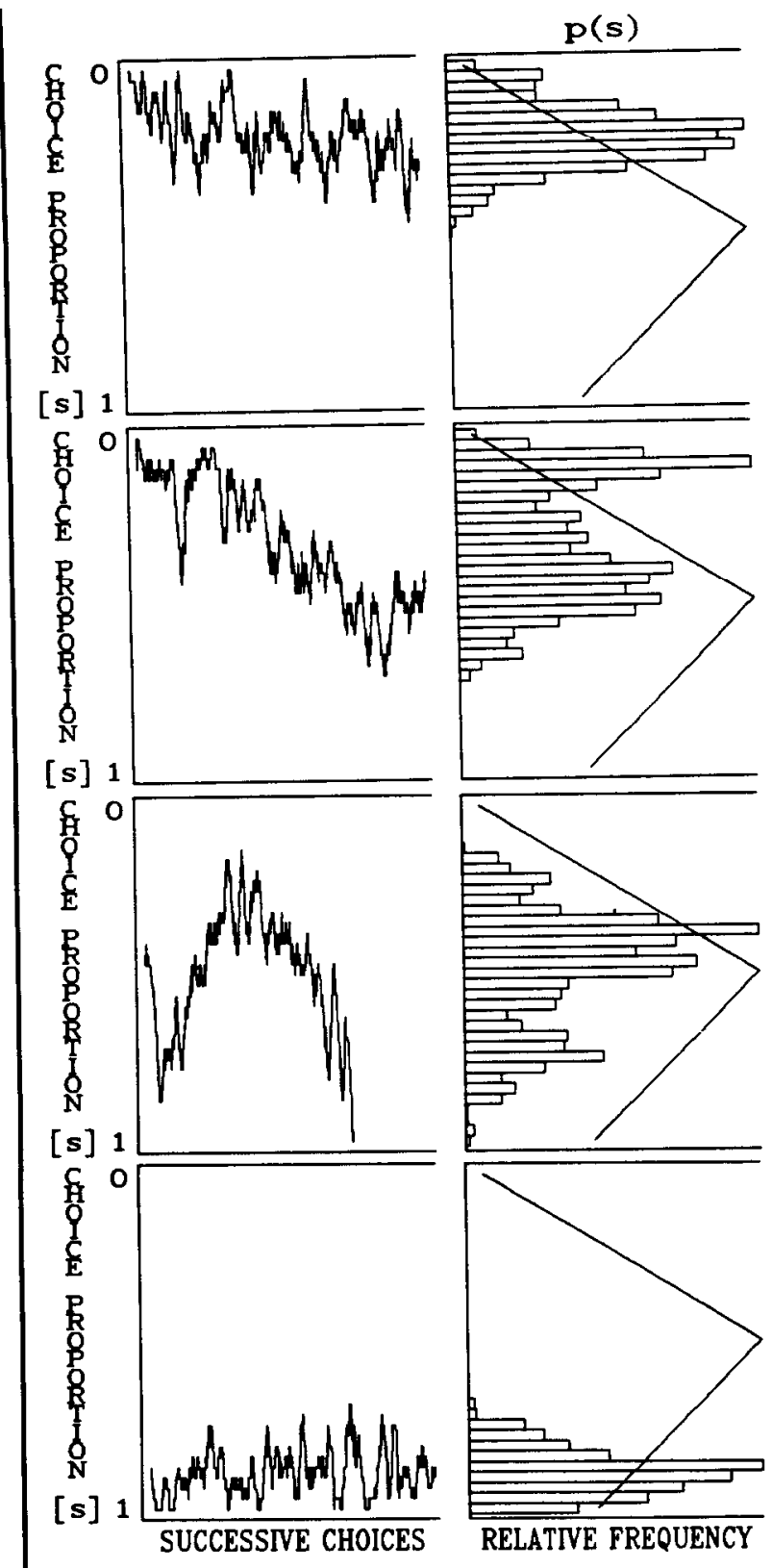

FIGURE 4. Four successive experimental sessions for pigeon 096 on the symmetrical interdependent schedule. Within each row, the left panel shows the choice trajectory throughout the session; the center panel shows the value of $s$ (averaged over the preceding 32 choices) choice-by-choice throughout the session; and the right-hand panel shows the distribution of s-values (choice distribution). The bilinear reward function is also shown. 


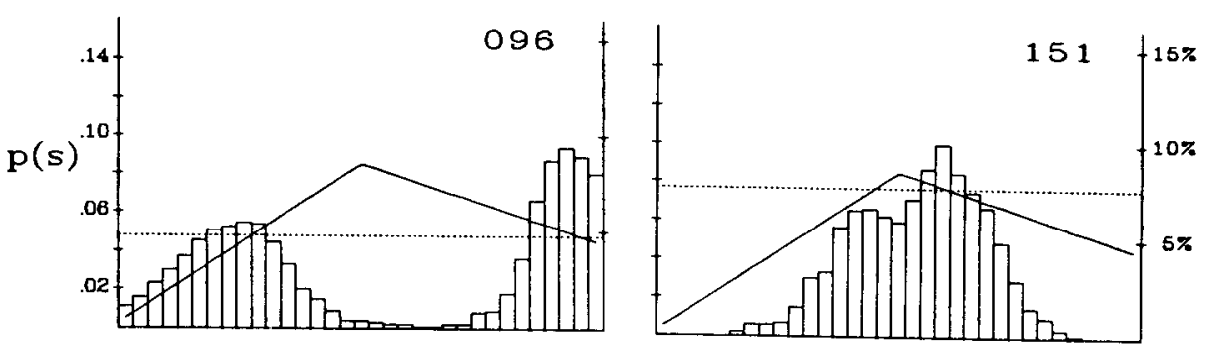

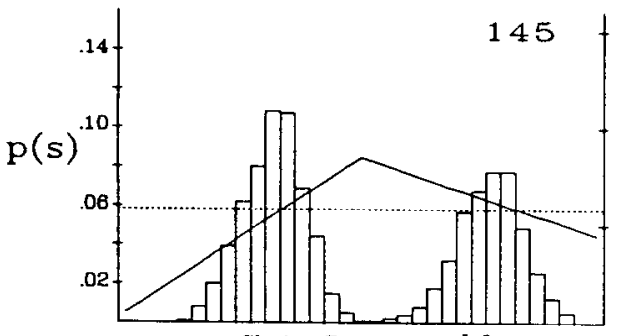

Choice Proportion [s]

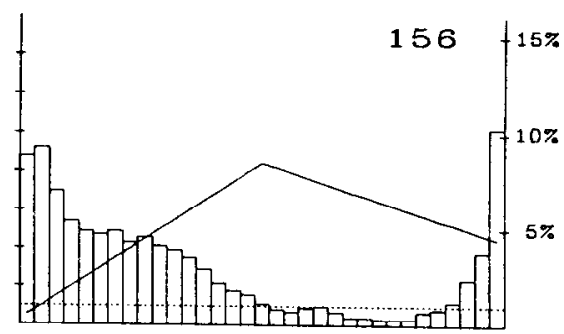

Choice Proportion [s]

FIGURE 5. Choice distributions (percentage of the total number of choices for which an animal achieved a particular proportion of right-hand choices, $s$, measured over the preceding 32 choices) on the symmetrical interdependent schedule for all four subjects. Data for each animal were combined from all 10 experimental days. Solid line is the bilinear reward function; broken line is at the estimated w-value for this choice distribution (see text).

TABLE 1. Choice modes and associated reward probabilities on the symmetrical interdependent schedule. Columns 2 and 3 give the s-values at each mode in Figure 5; columns 4 and 5 give the corresponding payoff probabilities, $p(s)$.

\begin{tabular}{c|c|c||c|c|} 
Bird & \multicolumn{2}{|c|}{ MODE : s } & $1^{\text {MODE }}: p(s)$ \\
& 1 & 2 & 1 & 2 \\
\hline 096 & .250 & .938 & .045 & .050 \\
145 & .313 & .797 & .055 & .062 \\
151 & .438 & .594 & .075 & .078 \\
156 & .031 & 1.000 & .010 & .045 \\
\hline
\end{tabular}

\section{DISCUSSION}

The symmetrical interdependent schedule forces matching at all choice proportions, so matching (melioration) makes no predictions here. Momentary maximizing is also inapplicable, because reward probability, $p(s)$, is always the same for both alternatives.

Bush-Mosteller predicts indifference, because the reward probabilities were the same on both keys (see Appendix A). Molar maximizing also implies indifference in 
this experiment, because our reward function peaked at $s=0.5$.

Despite its intuitive appeal, given that both keys always paid off with the same reward probability, no animal conformed to the prediction of indifference. The animal with the largest number of responses at the maximum reward probability, bird 151, nevertheless has distinctly fewer responses at indifference than at the two preferred choice proportions.

Despite some individual variation, all animals in this experiment show choice patterns of the type predicted by ratio invariance: animals 096, 151, and 145 show pattern $w_{2}$ in Figure 3; and bird 156 shows pattern $w_{3}$. The bilinear reward function, and a horizontal dashed line at the estimated (average of partialpreference modes) w-value, are superimposed on each choice distribution in Figure 5; comparison with Figure 3 shows how each distribution conforms to one of the three permissible patterns.

Each animal's "preferred" probability of reward (choice mode) gives us an estimate of its effect ratio, w. The partial-preference modes in Table 1 are therefore an estimate of $w$ for each animal. It is worth noting that the data are consistent with some tendency for w to increase with absolute reward probability; Table 1 shows that for the three birds where a comparison is possible (birds 096, 145 and 151), the preferred $\mathrm{p}(\mathrm{s})$ on the right-hand side of the maximum is always greater than the $\mathrm{p}(\mathrm{s})$ for the mode on the other side. Given normal variation in $s$ around the modal value, and the steeper slope of the left limb of the bilinear reward function, it is obvious that the average $p(s)$ on the right limb is likely to be $s$ light ly greater than the average $p(s)$ on the left limb. This small difference may account for the sma11, systematic difference between the two modal $\mathrm{p}(\mathrm{s})$ values.

We have presented data for only one reward function in Experiment 1. These data represent on $1 y$ a small selection from a large number of experiments that we have done with the SI procedure. For example, in other experiments we have looked at bilinear reward functions with modes at other than the point $s=0.5$ : under these conditions we have obtained instances of all three of the patterns permitted by reward invariance--including distributions with a mode at the indifference point, which is not the s-value that maximizes reward rate under these conditions. All these data (some of which we expect to report elsewhere) support ratioinvariance.

In this experiment we compared directly the predictions of ratio invariance and molar maximization (melioration and momentary maximizing made no point predictions). In Experiment 2, we compare directly the predictions of all four theories: maximization, melioration, momentary maximizing, and ratio invariance. 
EXPERIMENT 2 - Preference Patterns on an Asymetrical Interdependent Schedule

Experiment 1 showed that ratio invariance can explain data inconsistent with molar maximizing and not explained by (though consistent with) momentary maximizing and matching/melioration. In this experiment we describe a situation in which nondegenerate matching is precluded, and momentary maximizing, ratio invariance, and molar maximizing all make different predictions.

In Experiment 1 the probability of reward on the alternatives was always equal. Our ratio-invariance result might, therefore, be interpreted as a secondary process to which the animal "defaults" when the primary processes--melioration or momentary maximizing, perhaps--cannot operate. The asymmetrical-interdependent (AI) schedule provides a way to differentiate among these possibilities.

The AI schedule is similar to the symmetrical interdependent (SI) schedule in that the probability of reward for both alternatives changes depending on the animal's current preference. The AI schedule is different from the SI in that the probability of reward on the two alternatives is not the same. In the present experiment, a response to one alternative is always twice as likely to be rewarded as a response to the other. Moreover, the reward function is arranged so that choice proportions that favor the lower probability alternative have the highest overall probability of reward.

The top panel of Figure 6 shows the reward functions used in the first condition of this experiment. The reward probability for responses to one key (the Left, in the first condition), $q(s)$, is always twice that for responses to the Right key, $p(s): q(s)=2 p(s)$. We term the key with the higher reward probability the majority key (i.e., Left, in the first condition). The probability of reward for both alternatives increases linearly from close to zero at exclusive responding on the majority alternative to a maximum at exclusive responding on the minority alternative: $\mathrm{p}(\mathrm{s})=\mathrm{Ks}+\mathrm{J}$, where $\mathrm{K}=0.066$ and $\mathrm{J}$ is negligibly small $(0.001)$ in this experiment. Thus, the highest overall probability of reward (i.e., $s p(s)+(1-s) q(s)$ : broken line in the top pane1) is achieved by favoring the minority alternative almost exclusively. ${ }^{2}$

Because the AI schedule forces a deviation from matching (the schedule ensures that $q(s)=2 p(s)$, whereas matching requires $p(s)=q(s)$ ), the only way in which an animal can match is by exclusive responding on one of the alternatives (i.e., degenerate matching). Since melioration predicts that the animal will continually increment the proportion of responses made to the alternative with the higher local reward rate, exclusive choice of the majority key is predicted, which is also the momentary-maximizing prediction. Finally, unconstrained molar

2. For the functions given, the overall reward probability, $P$, is $P=s K s+$ $(1-8) 2 \mathrm{Ks}=2 \mathrm{Ks}-\mathrm{Ks}^{2}$, which has a maximum at $\mathrm{s}=1$, exclusive choice of the minority . 


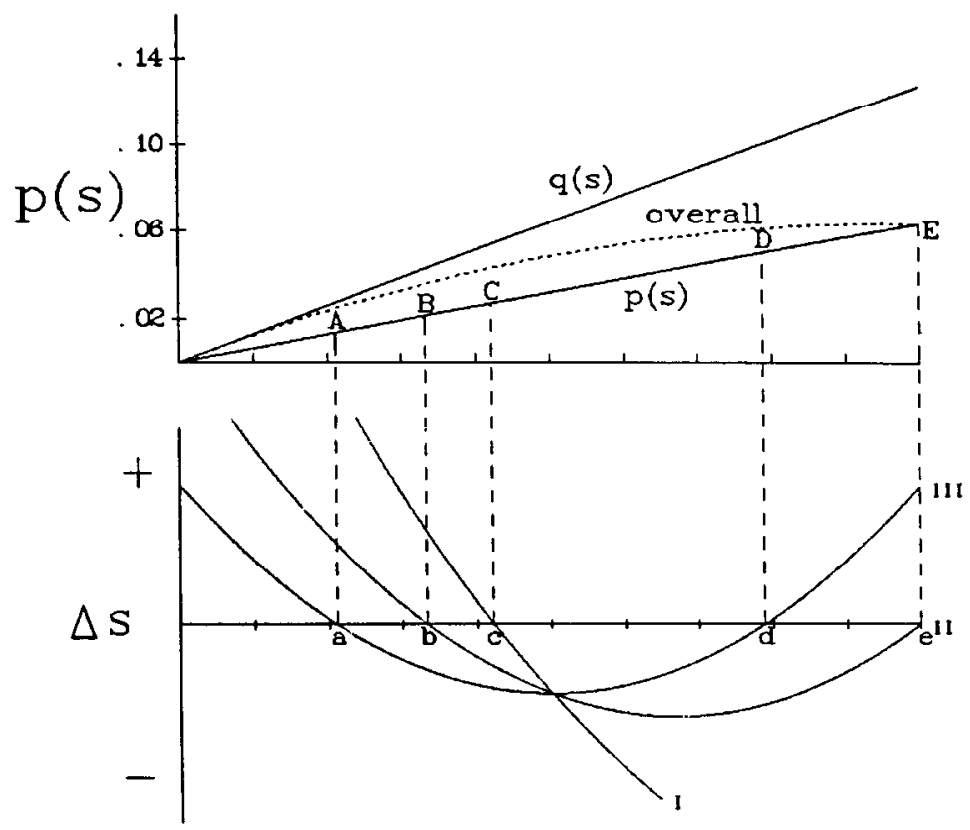

Choice Proportion [s]

FIGURE 6. Top pane1: Reward functions for the asymmetrical interdependent schedule in the positive-slope condition. The figure shows the probability of reward for the left-hand, $q(s)$, and right-hand, $p(s)$, alternatives as a function of the proportion of choices to the right-hand alternative, s: $q(s)=2 p(s)$, for all values of $s$. The dashed line is the overall probability of reward (i.e., $s p(s)+$ $(1-s) q(s))$. Bottom pane1: predictions of ratio-invariance for the asymmetrical interdependent schedule (positive-slope condition), for three values of $w / K(K=$ reward-function slope). The figure plots delta(s) as a function of $s$, calculated from Eq. 4 in the text by substituting the reward functions in Figure 6 for $p(s)$ and $q(\mathrm{~s})(\mathrm{Eq} . \mathrm{B} 7)$ : curve $\mathrm{I}, \mathrm{w} / \mathrm{K}=0.5 ; \mathrm{II}, \mathrm{w} / \mathrm{K}=1.0 ; \mathrm{III}, \mathrm{w} / \mathrm{K}=2.0$.

maximization predicts exclusive choice of the minority alternative.

Ratio-invariance predicts something different from both these extreme outcomes (see Appendix $B$ for the full argument). The animal should always develop a stable partial preference that favors the majority alternative (i.e., a preference on the side of indifference away from the maximizing solution: a mode in the region $s<$ $1 / 2$, for the positive-slope condition). When the partial-preference choice mode is at low $s$-values ( $\hat{\mathrm{s}}<1 / 3$, in the positive-slope condition) there is the possibility of a second preference mode at exclusive choice of the minority alternative.

The bottom panel of Figure 6 illustrates these predictions by substituting the reward functions in the top panel (i.e., $p(s)$ and $q(s)$ ) in Eq. 4, simplifying, 
then plotting delta(s) for the full range of s-values (Eq. B6). The bottom panel shows three delta(s) functions: for $\mathrm{w} / \mathrm{K}=.05,1.0$ and 2 (curves I, II and III). For values of $w / K>1$ (i.e., effect ratio greater than reward-function slope), there is a single stable equilibrium at a partial preference which favors the majority alternative ( $<1 / 2$, when left is the majority): line $I$ in Figure 6. When $w / K<1$, there is a possibility of a second choice mode at exclusive minority choice ( $s=1$, if right is the minority), and the partial-preference mode shifts further towards the majority $(s=0)$; line III in tle figure is an example. Line II defines the boundary between these two cases (i.e., $w / K=1$ ). In the terms we used earlier in discussing Figure 3, points $A, B$, and $C$ are attractors and point $D$ is unstable.

Thus, matching and momentary maximizing, molar maximizing, and ratio invariance make three distinct predictions about preference on the asymmetrical interdependent schedule, which is therefore a good way to choose among them.

METHOD

\section{Subjects}

Four adult White Carneau pigeons served. Each bird was maintained at $85 \%$ of its free-feeding weight, and all had previous experience with various schedules of reinforcement.

\section{Apparatus}

The apparatus and controlling instrumentation were the same as in Experiment 1.

\section{Procedure}

This procedure was based on the two-response, choice procedure described in Experiment 1. As in the SI schedule, the probability of reward for either of the two keys is determined by the proportion of choices over the last 32 cycles $(M=$ 32). But in the AI schedule the probability of reward on the two alternatives is not the same. In this experiment, the probability of reward was always twice as high on one alternative as on the other. For the minority choice $p(s)=0.066 \mathrm{~s}$ (so that $q(s)=0.132 \mathrm{~s}$ ). As before, the computer recalculated choice proportion after every response and determined reward probability for that response accordingly.

The first 50 choices in every experimental session were never rewarded. Sessions were terminated after the $48 \mathrm{th}$ reward.

For the first 10 sessions, the right key was the minority (positive-slope condition); for the last ten sessions, the significance of the two keys was reversed (negative-slope condition).

\section{RESULTS}

Figure 7 shows the choice distribution for the positive-slope condition. Figure 8 shows the same data for the negative-slope condition. For all birds in both conditions there is a partial-preference mode. For two of the birds in the positive-slope condition, 151 and 156 , there is also a mode at exclusive minority choice (156 shows a small partia1-preference mode near the minority in the 

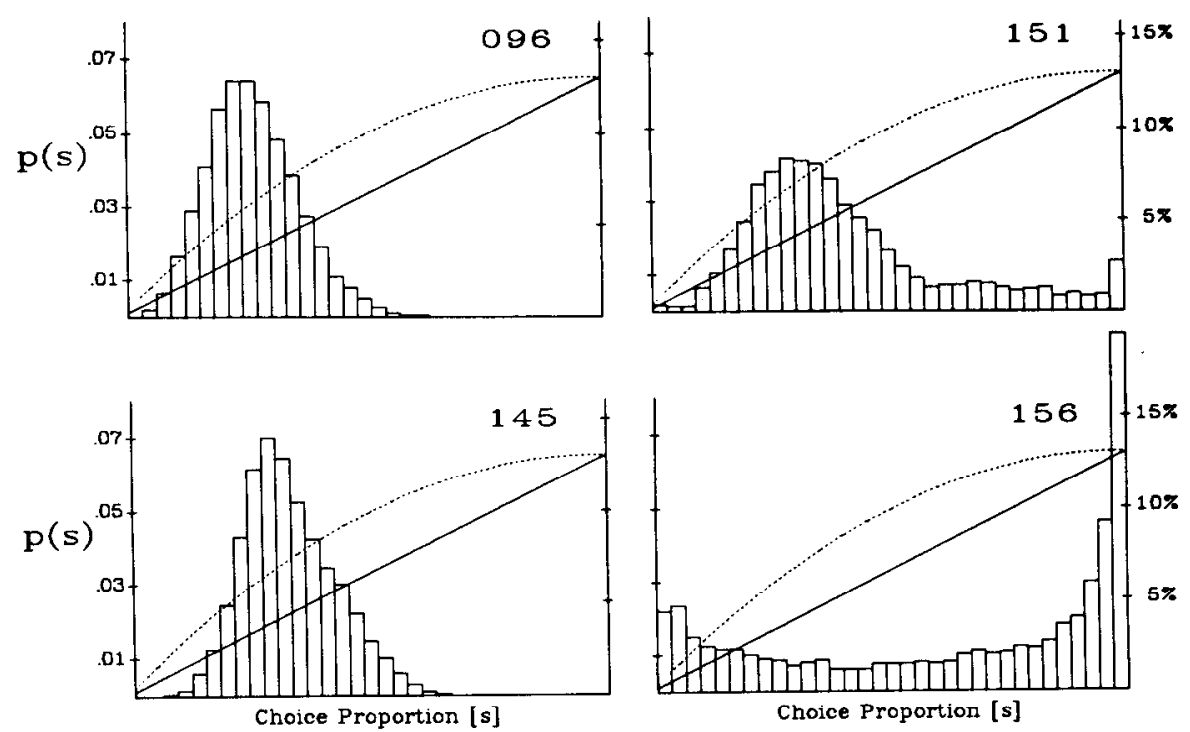

FIGURE 7. Choice distributions (percentage of the total number of choices for which an animal achieved a particular proportion of right-hand choices, $s$, measured over the preceding 32 choices) on the asymmetrical interdependent schedule. This figure shows the choice distributions for all animals for the positive-slope condition. Data for each animal were combined from all 10 experimental days in each condition. Solid line, $p(s)$ function; broken line, overall reward probability.
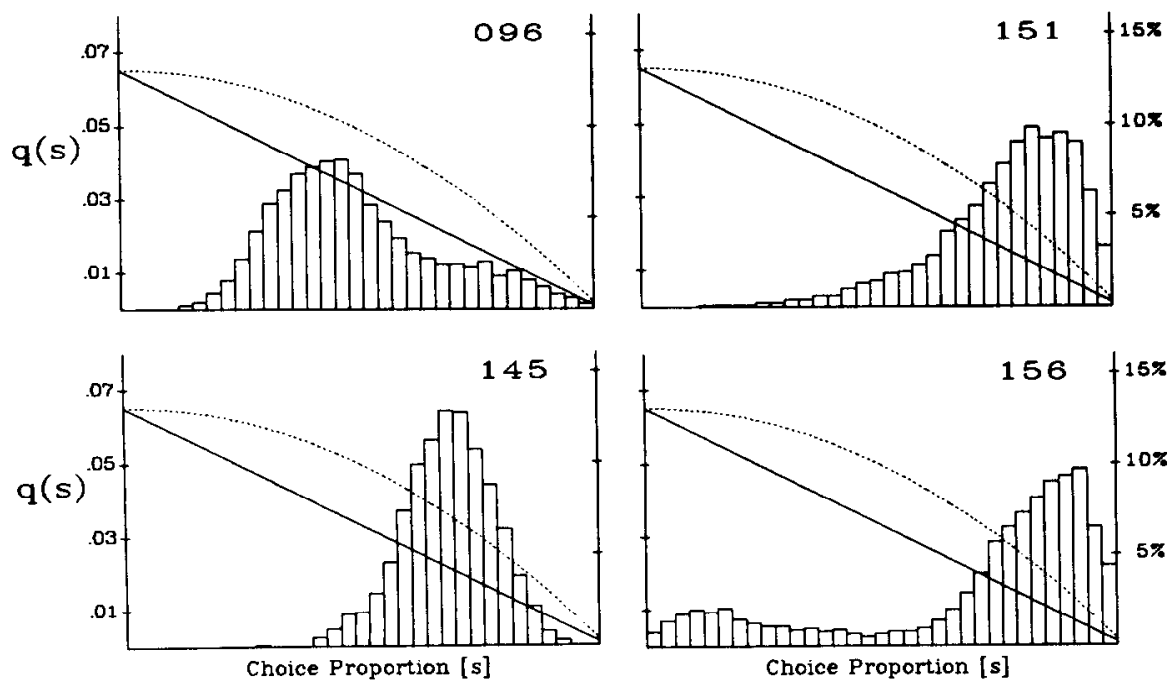

FIGURE 8. Figure 8 show the distributions for the negative-slope condition. Data for each animal were combined from all 10 experimental days in each condition. Solid line, $\mathrm{p}(\mathrm{s})$ function; broken line, overall reward probability. 
TABLE 2. Choice modes and associated reward probabilities for both conditions of the asymmetrical interdependent schedule. Columns 2 and 3 give the s-values at the partial-preference choice mode and the corresponding reward probability, $p$ (s). Column 4 is the walue calculated from the partial-preference mode, from Eq. B6: reward-function slope, $K$, was 0.066 .

\section{Positive-Slope Condition}

\begin{tabular}{l|c|c|c|} 
Bird & $\mathrm{s}$ & $\mathrm{p}(\mathrm{s})$ & $\begin{array}{c}\text { Calculated } \\
\mathrm{w}\end{array}$ \\
\hline 096 & & & .039 \\
145 & .235 & .016 & .050 \\
151 & .281 & .019 & .050 \\
156 & .281 & .019 & .006 \\
\hline
\end{tabular}

Negative-slope Condition

\begin{tabular}{r|r|r|r|}
096 & .469 & .070 & -.230 \\
145 & .688 & .042 & .059 \\
151 & .844 & .022 & .025 \\
156 & .938 & .010 & .010 \\
\hline
\end{tabular}

negative slope-condition, but a much larger partial-preference mode on the majority side). Most importantly, all animals, except 096 in the negative-slope condition (Figure 8), show a predominant partial-preference mode on the majority side.

Table 2 gives the choice proportion and the probability of reward for both alternatives at the partial-preference mode for the positive-slope and negativeslope conditions. Table 2 also gives the walues for each animal, calculated using Equation B6.

\section{DISCUSSION}

Neither matching, nor momentary maximizing, nor molar maximizing predicts the results from this experiment. Both matching and momentary maximizing predict exclusive choice of the majority alternative, which did not occur. Molar maximizing implies simple exclusive choice of the minority alternative, which also failed to occur. Birds 151 and 156 do show a choice mode at the minority alternative, but this preference is in conjunction with the stable partialpreference mode near the majority alternative, consistent with that shown by the other animals. Molar maximizing cannot account for both aspects of these results.

The results in seven out of eight subject-conditions are consistent with ratioinvariance: (a) All animals showed a stable partial preference that favored the majority alternative in the positive-slope condition. (b) All of the animals, except bird 096, showed a stable partial preference that favored the majority 
alternative in the negative-slope condition. (c) Two of the animals, birds 151 and 156, showed a second mode at exclusive choice of the minority alternative in the positive-slope condition, which is also consistent with ratio invariance.

Bird 096's performance in the negative-slope condition cannot be accounted for by ratio-invariance (note the negative calculated w-value for this animal in Table 2 ), and the small secondary partial-preference mode shown by bird 156 in the negative-slope condition is also a weak exception. Later studies using the AI procedure with bird 096 suggest that 10 sessions may simply have been too short a time for its behavior to stabilize in the first condition of this experiment; similar consideration may apply to bird 156-its second mode in the negative-slope condition may be a vestige of the previous condition. Since we lack detailed information on the reward function $a(s)$ (and the related nonreward function, $b(s)$ ), we have no way of predicting the time it will take for the reward-following process to stabilize.

Some of the birds showed a second mode at exclusive choice of the minority. Since all showed calculated w-values less than $\mathrm{K}$ (see Table 2), this is also consistent with ratio invariance (see Appendix $B$ ).

We had expected that by giving two reward functions that were mirror images we could recover w values that were the same for each animal in both conditions. Pigeons 145 and 156 lived up to expectations, whereas bird 151 did not. Because the performance of bird 096 in the negative-slope condition is inconsistent with ratio-invariance, it is not possible to compare $w$ values in the two conditions for this animal.

The asymmetrical interdependent procedure shows the dangers of pure hillclimbing choice rules, like melioration and momentary maximizing. Both rules, by always selecting the better of two alternatives, can under many conditions drive the animal towards extinction. A rule that takes account of absolute, as well as relative, payoff cannot fall into this kind of trap. Ratio invariance is just such a rule, with the effect ratio, w, taking account of absolute payoff probability. No matter how good an alternative in relative terms, if it pays of with a probability less than w, ratio invariance will force a shift to other alternatives. Ratio invariance may be summarized as "if it's good enough, go for it; otherwise, sample."

\section{GENERAL DISCUSSION}

If one choice has a higher probability of being rewarded-or is rewarded at a higher rate- than another, an animal would do well to opt for it. Payoff probability and reward rate should be important determiners of choice. Numerous studies (see Mackintosh, 1974; Staddon, 1983, for reviews) show that animals do 
indeed choose the alternative with the higher probability or rate of reward under many conditions. Thus, it has seemed reasonable to assume that animals are directly sensitive to reward probability and reward rate.

Although the choice of the highest rate or highest probability alternative may be adaptive, the strategy that accomplishes this may be neither direct nor infallible. It has become a truism in behavioral ecology that animals often act intelligently using unintelligent means. No doubt human beings are equally unsophisticated under many conditions. Hence, we might expect organisms to identify the higher payoff option using a simple strategy that works well under many natural conditions, but which nevertheless may be induced to fail under some artificial conditions. The nature of these failures can tell us something about the nature of the strategy. Our results show that apparent sensitivity to reward probability, and perhaps reward rate as well, is a consequence of something simpler: an effect-ratio-invariant reward-following strategy--ratio invariance-at least under the restricted conditions we have studied.

In addition to predicting in some detail the complex results from our experiments, ratio invariance has several interesting properties. For example, that reward (reinforcement) reduces behavioral variation has often been noted (e.g., Schwartz, 1980; Staddon \& Simme1hag, 1971). The suggestion has even been made that the reduction of variation is the defining property of reinforcement. It is noteworthy, therefore, that ratio-invariance provides a mechanism that has just this property. Our pilot experiments showed that pigeons are more willing to fixate on one key when the reward probability is high than when it is low; in Experiments 1 and 2, the higher the reward probability (relative to w), the closer the choice modes to exclusivity. Effect-ratio invariance provides an explanation for the covariation of stereotypy and reward probability.

Ratio-invariant reward-following provides both an old and a new account of recurrent choice. It is old because it is another stochastic "learning" (really performance) model. It is new in that it assumes source independence (i.e., dependence of the sign, but not the absolute amount, of choice-probability change on the source of reward or nonreward) and constancy of the effect ratio--neither of which is true of standard linear-operator stochastic models. Unlike some stochastic models (e.8., Rescorla \& Wagner, 1972; Vaughan, 1982) it takes as dependent variable choice proportion, not hard-to-measure "response strength" or "stimulus value," which is a pleasing gain in concreteness. Moreover, our experimental results seem absolutely incompatible with the simple Bush-Mosteller approach as well as being inexplicable by current theories of free-operant choice, such as molar and momentary maximizing and melioration.

An intriguing property of ratio invariance is the effect ratio $w$ (the effect of nonreward relative to the sum of the effects of reward and nonreward), which seems 
to be approximately constant for a given animal in a given situation. We might expect that conditions (such as depression) or treatments (such as food deprivation or drugs) that affect sensitivity to reward will have measurable effects on w.

If $w$ increases with overall reward rate, as our earlier arguments suggest it might, then ratio invariance has precisely the properties needed for an optimalpatch-foraging mechanism consistent with the much-studied marginal-value theorem (Charnov, 1976). The argument is as follows. As a given food patch depletes, the reward probability must drop, eventually reaching a $p$ value less than the value of w. At this point, the animal should begin to show a partial preference (as in Experiment 1), i.e., to leave the patch and sample another. If $w$ is directly related to overall food rate, as seems likely, then this "giving-up time" will be shorter (i.e., at higher probability values) in richer environments, exactly as the marginal-value theorem requires.

Ratio invariance has properties similar to what Simon (1956) has termed satisficing, that is, it yields behavior that is often, but not always optimal-but always results in some gain. An organism that satisfices will settle for an acceptable alternative, even if a better one is available, and will not settle for the best alternative if it is not good enough. Both of these characteristics are predicted by ratio invariance in the two-armed-bandit situation since fixation on the minority alternative is possible, providing the minority payoff probability is greater than $w$; and partial preference is possible if neither p nor $q$ exceeds $w$.

Neither melioration nor momentary maximizing are satioficing rules. In Experiment 2, for example, either rule, strictly applied, leads to a reward rate close to zero-because both involve comparison only between proferred alternatives, not with an internal standard. The essence of satisficing is the acceptance or rejection of alternative with respect to a threshold value (parameter $w$, in ratio-invariance). This property is guaranteed by our version of reward-following because it includes nonreward avoidance. Not only does the probability of a behavior increase following reward, it also decreases following nonreward-which protects the animal against the self-generated extinction entailed by both melioration and momentary maximizing under the asymmetrical interdependent procedure in Expcriment 2.

\section{Extensions to other choice procedures}

Two-armed bandit: unequal probabilities. Exclusive choice of the majority is the usual result on these procedures (e.g., Herrnstein \& Loveland, 1975), and it is the prediction of reward invariance when $p<w<q$ (Appendix A). Ratio invariance also makes the conter-intuitive prediction that animals can show exclusive choice for the minority alternative when the subject begins with that 
initial preference and $p$ and $q$ are both greater than w (see Appendix A for proof), and we have found (Horner \& Staddon, unpublished data) that animals will often favor the minority alternative in this procedure for some time after a schedule change, if they begin with that bias. We have not found a persistent minority preference, however.

The transience of preference for the minority seems to depend on the nature of the effect functions $a(s)$ and $b(s)$ and the randomness of the two-arm-bandit procedure. The randomness of the reward schedules will occasionally cause the subject to experience a run of nonrewards on the minority side sufficient (for many $a(s)$ and $b(s)$ functions) to produce a significant shift towards the majority, where responding may be "captured." Table 3 illustrates this for the simplest functions $(a(s)=$ constant, and $b(s)=$ constant, with the program limiting s to the interval $0-1$ ) by means of a simulation in which 1000 "stat" subjects were run, each with an initial preference for the minority. The first effect is increased concentration at the minority, but after many choices all preferences shift to the majority. Similar simulations using the effect functions $a(s)=$ $A s(1-s)$ and $b(s)=B s(1-s)$, where $0<B<A<1$, show that persistent exclusive minority choice is possible for many values of $A$ and $B$, however, so that exclusive minority choice obviously depends on the nature of the effect functions. ${ }^{3}$

This apparent inconsistency between prediction and data in the two-armed-bandit situation emphasizes the incompleteness of reward invariance as a theory. Our stability analysis leaves out of account the rate at which changes occur (because we do not know the details of $a(s)$ and $b(s)$, the reward and nonreward effects). Hence, we can predict only choice proportions (s-values) where there can be modes; we cannot predict the relative sizes of observed modes. Nevertheless, it is important to realize that these predictions are quite strong; ratio invariance would be absolutely invalidated if stable modes occurred at other places (even though absence of a mode at a permitted s-value is not sufficient to disprove the mode1). We cannot with assurance define just what we mean by stable but this is not a serious limitation in practice, since animals usually adjust to the procedures we have been using within a reasonable time.

Moreover, by separating out the dynamic aspects of choice, which depend upon $a(s)$ and $b(s)$, from the stability properties, which depend only on their ratio (i.e., on the effect ratio $w$ ), we pave the way for a truly comprehensive, dynamic account.

3. For the first simulation, $a(s)=0.098$ and $b(s)=0.002$, with s-values< 0 or $>1$ set equal to 0 or 1 , respectively, so that $s$ is limited to the interval $0-1$. For the second simulation, $A=4 \mathrm{a}$ and $B=4 \mathrm{~b}$. We have not pursued these simulations to the point where we can be sure whether the exclusive minority choice under the second pair of effect functions is truly permanent, or merely relatively long lasting. 
TABLE 3. Ratio-invariant reward-following simulation for choice between two unequal probabilistic schedules. The table gives the percentage (out of 1000 "stat" subjects) showing different values of $s$ (columns) after different numbers of choices, from 1 to 10,000 (rows). Al1 subjects began with s in the interval 0.65-0.73. The reward probabilities were $p=0.05$ and $q=0.10 ; w=0.02$, and $a(s)$ and $b(s)$ were positive constants over the range $0<s<1$, zero otherwise. Zeroes are omitted to increase readability.

Mean Choice Proportion (s)

\begin{tabular}{r||r|r|r|r|r|r|r|r|r|r|r|} 
No. of resp. & $0-.09$ & -.18 & -.27 & -.36 & -.45 & -.55 & -.64 & -.73 & -.82 & -.91 & -1.0 \\
\hline 1 & & & & & & & & 100 & & & \\
5 & & & & & & & 6 & 83 & 9 & & \\
20 & & & & & 1 & 5 & 19 & 39 & 21 & 9 & 2 \\
50 & & & 2 & 6 & 14 & 16 & 15 & 17 & 15 \\
100 & 7 & 3 & 4 & 5 & 7 & 8 & 8 & 9 & 15 & 23 \\
200 & 29 & 4 & 2 & 2 & 2 & 3 & 3 & 3 & 4 & 14 & 29 \\
500 & 48 & 3 & & 1 & & & 1 & 2 & 3 & 12 & 25 \\
1000 & 58 & 5 & & & & & 1 & 1 & 2 & 8 & 20 \\
2000 & 76 & 4 & & & & & & & 1 & 5 & 9 \\
5000 & 88 & 8 & & & & & & & & & 1 \\
10000 & 90 & 8 & & & & & & & &
\end{tabular}

Interdependent variable-interval schedules. Our analysis may account for regularities in the data of Herrnstein and Vaughan (1980) on an interdependent procedure based on a variable-interval (VI) schedule. In this experiment, local rates of reward were held constant and equal for both choices, but the rate depended upon current preference (averaged over a block of time). The reward function had a maximum at $s=0.25$; nevertheless, all four pigeons showed a preference close to indifference ( $s=0.5$ : Herrnstein \& Vaughan, Fig. 5.9).

Ratio-invariance may account for these data as follows: On variable-interval schedules, response rate is typically much higher than reward rate. For example, pigeons may peck at $80 / \mathrm{min}$ for rewards delivered at a rate of just $1 / \mathrm{min}$ (cf. Catania \& Reynolds, 1968), so that reward probability, $p(s)$, is typically quite small (on the order of 0.01-0.02), smaller than the typical walues in our experiments. When the reward function in an equal-probability interdependent schedule does not permit a $p(s)$ value $\geq w$, ratio invariance predicts indifference between the alternatives (see Appendix B), which is what Herrnstein and Vaughan found.

Position habits. It is well known that animals in discrimination tasks with spatial choice responses (e.g., the left and right arms of a $\mathrm{T}$-maze) will often revert to fixed choice for one alternative when they are unable to make the necessary discrimination. The reward probabilities in these experiments are typically high. It may be possible to obtain a reward on every trial if responding is correct, for example, which implies $p=0.5$ even if the animal cannot discriminate at all. Hence, position habits may simply reflect ratioinvariance, as we showed in Experiment 1 for the high-reward-probability 
condition. An implication of this analysis is that position habits shown repeatedly by the same individual in a series of difficult tasks, such as successive discrimination reversals, should be uncorrelated from one task to the next (i.e., the animal should settle randomly on one side or another). Apparently this kind of independence is often found (N. J. Mackintosh, personal communication), although we have not been able to find published data bearing on this prediction.

Concurrent variable-interval schedules. Ratio-invariance can be applied to choice between variable-interval schedules, a much-studied procedure, if we make some assumption about the way in which reward rate affects response rate; time, excluded heretofore, must be reintroduced into the analysis. Nevertheless, some interesting predictions can be derived using only the well-established fact that on VI schedules reward rate has little effect on response rate, which is approximately constant over a wide range of VI values (cf. Catania \& Reynolds, 1968).

On VI schedules, reward probability is inversely related to response rate--the more slowly the pigeon pecks, the more likely each peck will be rewarded. The exact form of this inverse relation depends upon the molar feedback function (Baum, 1973) we assume for VI. If we let response rate on the two choices be constant, $x+y=k$, and use the feedback function appropriate for random responding (Staddon \& Motheral, 1978), then the reward probabilities are: $p(s)=$ $R(x) / s=A /(A+x)$ and $p(1-s)=B /(B+y)$, where $A$ and $B$ are the $s c h e d u l e d$ maximum reward rates for the two VI schedules, and $s(=x /(x+y)$, where $x$ and $y$ are response rates to the two alternatives) is the proportion of right-choices, as before. Substituting these values for $p(s)$ and $p(1-s)$ for $p(s)$ and $q(s)$ in equation 4 allows us to derive the delta(s) function for the two-choice VI schedule (see Appendix $\mathrm{C}$ ).

Ratio-invariance makes two main predictions about choice between VI schedules. First, as the schedules becomes richer, the model implies exclusive choice-because typical p values are high; conversely, indifference is implied between lean choices. This prediction is consistent with the data of Fantino et al. (1972), who showed that animals tended toward exclusive choice on the majority alternative for very rich VI schedules (choice between VI $6 \mathrm{~s}$ and VI 12s) and were more indifferent between lean schedules (VI 600s vs. VI 1200s) than between moderate schedules (VI 60s vs. VI120s). (Note that these changes in preference had little effect on matching, because of the properties of the VI molar feedback function; response ratios continue to match obtained reward ratios, but not necessarily scheduled reward ratios: cf. Staddon, Hinson \& Kram, 1981.)

A related prediction of ratio-invariance involves the performance bias of individual animals. Given that overall response rate is approximately constant 
over a wide range of scheduled VI values, ratio invariance predicts consistent overmatching or undermatching with respect to scheduled relative reward rates for different values of $w$. As the value of $w$ changes from 0.5 to 0 , the effect is to move from consistent undermatching to consistent overmatching with respect to scheduled values. Thus, manipulations that affect the value of $w$ (such as food deprivation and absolute food rate, for example) should have corresponding effects on the degree of overmatching or undermatching. Very hungry animals should overmatch, satiated animals should undermatch, on typical concurrent vi schedules. This second prediction has not been systematically tested.

Both of these predictions assume that the changes in reward probability associated with different VI values have a larger effect than any opposing change in w. We suspect, for reasons already discussed, that $w$ will increase as reward rate increases (i.e., the differential effect of reward will be reduced at high reward rates). Our predictions assume that the increase in reward probability, p, associated with richer VI values will be such as to produce a net increase in the ratio $\mathrm{p} / \mathrm{w}$, even though $\mathrm{w}$ also increases. Obviously, definitive extension of our analysis to VI schedules must await measurement of the effects of reward rate and absolute reward probability on the value of $w$.

\section{CONCLUSION}

We have shown that a source-independent, effect-ratio-invariant, rewardfollowing process is sufficient to account for a wide range of individual-subject data on simple and interdependent probabilistic schedules, as well as published data on several standard choice procedures such as the two-armed bandit, and concurrent and interdependent variable-interval schedules. Ratio invariance explains results either not explained by, or actually incompatible with, standard treatments of choice in operant situations such as Bush-Mosteller, melioration (matching), and unconstrained molar or momentary maximizing. Ratio invariance is a type of satisficing and avoids the traps to which pure hill-climbing rules are liable, has properties consistent with optimal-foraging theory, and may underlie puzzling empirical effects such as position habits.

Ratio invariance may not be the, or the only, process operating, in more complex situations where cues such as time are available, however, such as choice between interval schedules-where processes such as momentary maximizing may dominate. Future work will need to settle on just how many processes are involved in simple recurrent choice, and whether they cooperate or act in a mutually exclusive fashion under various choice regimens. 


\section{ACKNOWLEDGEMENTS}

This paper is based on a dissertation submitted by JMH in partial fulfillment of the requirements for the Ph.D. degree at Duke University. Parts of the work were presented at the November, 1985, meeting of the Psychonomic Society in Boston, MA. We thank Juan Delius and Mark Rausher for comments on the manuscript and Donald Laming for suggesting the Bush-Mosteller stability analysis. The research was supported by grants to Duke University from the National Science Foundation and the Pew Memorial Trust. JERS wishes to thank the Alexander von Humboldt-Stiftung for support, and Juan Delius and the Ruhr-UniversitHt, Bochum, FRG, for hospitality during the preparation of the paper.

\section{REPERENCES}

Allison, J., 1983. Behavioral Economics. Praeger, New York.

Baum, W. M, 1973. The correlation-based law of effect. J. Exp. Anal. Behav., 20: $137-153$

Bush, R. R. and Mosteller, F., 1955. Stochastic Models for Learning. Wiley, New York.

Catania, A. C. and Reynolds, G. S., 1968. A quantitative analysis of the behavior maintained by interval schedules of reinforcement. J. Exp. Ana1. Behav., 11: 327-383.

Charnov, E. L., 1976. Optimal foraging: The marginal value theorem. Theor. Pop. Bio., 9: 129-136.

Daly, H. B. and Daly, J. T., 1982. A mathematical model of reward and aversive nonreward: Its application to over 30 appetitive learning situations. J. Exp. Peych.: Gen., 111:441-480.

Fantino, E., Squires, N., Delbrllck, N. and Peterson, C, 1972. Choice behavior and the accessibility of the reinforcer. J. Exp. Anal. Behav., 18: $35-43$.

Herrnstein, R. J, and Loveland, D. H., 1975. Maximizing and matching on concurrent ratio schedules. J. Exp. Ana1. Behav., 24: 107-116.

Herrnstein R. J. and Vaughan, W., 1980. Melioration and behavioral allocation. In: J. E. R. Staddon (Editor), Limits to Action: The Allocation of Individual Behavior. Academic Press, New York.

Hinson, J. M. and Staddon, J. E. R., 1983a. Hill-climbing by pigeons. J. Exp. Ana 1. Behav., 39: 25-47.

Hinson, J.H. and Staddon, J. E. R., 1983b. Matching, maximizing and hil1c1imbing. J. Exp. Ana1. Behav., 40:321-331.

Horner, J. M., 1986. Reward-following as a determinant of choice. Ph.D. Dissertation, Duke.

Mackintosh, N. J., 1974. The Psychology of Animal Learning. Academic Press, New York.

Mosteller, F., 1955. Stochastic Learning Models. Proceedings of the Third Berkeley Symposium on Mathematical Statistics, 5: 151-167.

Myerson, J. and Hale, S., 1984. Transition-State Behavior on Conc VR VR: A Comparison of Melioration and the Kinetic Model. Paper presented at the Meeting of the Association for Behavior Analysist, Nashville, TN.

Myerson, J. and Miezin, F. M., 1980. The kinetics of choice: An operant systems analysis. Psychol. Rev., 87: 160-174.

Rachlin, H., 1978. A molar theory of reinforcement schedules. J. Exp. Anal. Behav., 30:345-360.

Rachlin, H. and Burkhard, B., 1978. The temporal triangle: Response substitution in instrumental conditioning. Psycho1. Rev., 85: 22-48.

Rachlin, H., Green, L., Kage1, J. H. and Batta1io, R. C., 1976. Economic demand theory and psychological studies of choice. In: G. Bower (Editor), The Psychology of Learning and Motivation: Vo1. 10. Academic Press, New York.

Rachlin, H. C., Kage 1, J. H. and Battalio, R. C., 1980. Substitutability in time allocation. Psychol. Rev., 87: 355-374.

Rescorla, R. A. and Wagner, A. R., 1972. A theory of Pavlovian conditioning: Variations in the effectiveness of reinforcement and nonreinforcement. In: A. Black and W. R. Prokasy (Editors), Classical Conditioning II. AppletonCentury-Crofts, New York. 
Schelling, T. C., 1978. Micromotives and Macrobehavior. Norton, New York.

Schwartz, B., 1980. Development of complex, stereotyped behavior in the pigeon. J. Exp. Ana 1. Behav., 33: 153-166.

Shimp, C. P., 1966. Probabilistically reinforced choice behavior in pigeons. J. Exp. Ana 1. Behav., 9: 443-455.

Shimp, C. P., 1969. Optimal behavior in free-operant experiments. Psychol. Rev., $76: 97-112$.

Silberberg, A., Hamilton, B., Ziriax, J. M. and Casey, S., 1978. The structure of choice. J. Exp. Psych.: An. Behav. Proc., 4: 368-398.

Simon, H. A., 1956. Rational choice and the structure of the environment. Psychol. Rev., 63: 129-138.

Staddon, J. E. R., 1965. Some properties of spaced responding in pigeons. J. Exp. Ana1. Behav., 8: 19-27.

Staddon, J. E. R., 1977. On Herrnstein's equation and related forms. J. Exp. Ana]. Behav., 28: 163-170.

Staddon J. E. R., 1980. Optimality analyses of operant behavior and their relation to optimal foraging. In: J. E. R. Staddon (Editor), Limits to Action: The Allocation of Individual Behavior. Academic Press, New York.

Staddon J. E. R., 1983. Adaptive Behavior and Learning. Cambridge University Press, Cambridge.

Staddon, J. E. R, and Hinson, J. M, 1983. Optimization: A result or a mechanism? Science, 221: 976-977.

Staddon, J. E. R., Hinson, J. M. and Kram, R., 1981. Optimal choice. J. Exp. Ana 1. Behav., 35: 397-412.

Staddon J. E. R. and Motheral, S,, 1978. On matching and maximizing in operant choice experiments. Psycho1. Rev., 85: 436-444.

Staddon, J. E. R. and Simmelhag, V., 1971. The "superstition" experiment: A reexamination of its implications for the principles of adaptive behavior. Psycho1. Rev., 78: 3-43.

Sternberg, S., 1963. Stochastic learning theory. In: R. D. Luce, R. R. Bush and E. Galanter (Editors), Handbook of Mathematical Psychology: Vol. 2. Wiley, New York.

Vaughan, W., 1981. Melioration, matching and maximization. J. Exp. Ana 1. Behav., 36: $141-149$.

Vaughan, W., 1982. Choice and the Rescorla-Wagner model. In: M. L. Commons, R. J. Herrnstein and H. Rachlin (Editors), Quantitative Analyses of Behavior: Vo1. 2 , Matching and Maximizing Accounts. Ballinger, Cambridge, MA.

\section{APPENDIX A}

Stability Properties of Rerard-Following Models

Ratio invariance. We consider the general two-choice probabilistic situation, with payoff probabilities $p$ and $q$ on right and left, respectively. Let $s_{n}$ be the probability that the $n$th response is to the right-hand alternative. Then the four possible sequences of events consequent on the nth response, together with their associated probabilities, are as shown in the contingency table below (event probabilities are at the top in each cell, changes in s below in []);

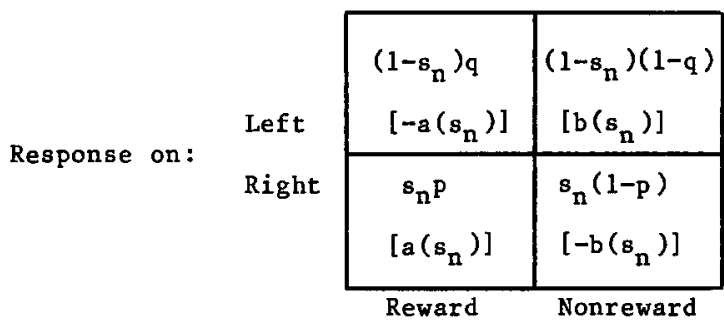

$$
\begin{aligned}
& \text { Outcome }
\end{aligned}
$$


(The changes in $s_{n}$ following reward and nonreward, $a\left(s_{n}\right)$ and $b\left(s_{n}\right)$, can be constrained such that $0<s_{n}<1$, but this is not necessary for stability analysis.) The four possible changes in $s_{n}$, i.e., $s_{n+1}-s_{n}$, are as follows:

Reward on R: $s_{n+1}-s_{n}=a\left(s_{n}\right)$ with probability $s_{n} p=a\left(s_{n}\right) s_{n}$ P

Nonr. on R: $s_{n+1}-s_{n}=-b\left(s_{n}\right)$ with probability $s_{n}(1-p)=-b\left(s_{n}\right) s_{n}(1-p)$

Reward on I: $s_{n+1}-s_{n}=-a\left(s_{n}\right)$ with probability $\left(1-s_{n}\right) q=-a\left(s_{n}\right)\left(1-s_{n}\right) q$

Nonr. on L: $\quad s_{n+1}-s_{n}=b\left(s_{n}\right)$ with probability $\left(1-s_{n}\right)(1-q)=b\left(s_{n}\right)\left(1-s_{n}\right)(1-q)$

The expected value of the change in $s$ following response $n$ can be obtained by summing the four contributions:

$$
E\left\{s_{n+1}-s_{n} \mid s_{n}\right\}=a\left(s_{n}\right) s_{n} p-b\left(s_{n}\right) s_{n}(1-p)-a\left(s_{n}\right)\left(1-s_{n}\right) q+b\left(s_{n}\right)\left(1-s_{n}\right)(1-q),
$$

which can be rearranged to

$$
E\left\{s_{n+1}-s_{n} \mid s_{n}\right\}=s_{n}\left[(p+q)\left(a\left(s_{n}\right)+b\left(s_{n}\right)\right)-2 b\left(s_{n}\right)\right]+b\left(s_{n}\right)-q\left(a\left(s_{n}\right)+b\left(s_{n}\right)\right),
$$

which is the same as Eq. 2 in the text, where $E\left\{s_{n+1}-s_{n} \mid s_{n}\right\}$ is termed delta(s) and $s_{n}$ is written simply as $s$; equation 3 ,

$$
\operatorname{delta}(s)=s(p+q-2 w)+w-q,
$$

where $w=b(s) /[a(s)+b(s)]$, is there derived from Eq.2.

Two-armed bandit: stability analysis. For ease of reading in subsequent analyses we replace $s$ by $s$. Equation 3 in the text describes the twoarm-bandit situation, with payof probabilities $p$ and $q$. The equilibrium behavior for ratio invariance depends upon the conditions for equilibrium (delta $(s)=0)$, partial preference $(0<\hat{\mathbf{s}}<1)$, and stability (negative slope for equation 3 ).

The equilibrium condition yields

$$
\hat{s}=(q-w) /(p+q-2 w),
$$

which gives $\hat{\mathbf{s}}=0.5$ for the case $p=q$.

The stability condition requires that $p+q<2 w$, which is stable for the case $p=q$ if $p<w$. For a partial preference, given that $\hat{s}$ must always be positive, the numerator of Eq. Al must be negative if the partial preference is to be stable, i.e., $w>q$, so that the condition for a stable partial preference is that $p, q<w$.

If $p+q>2 w$, so that there is no stable equilibrium, we can distinguish two cases: $p, q>w$, or $p>w>q$ (or $q<w<p)$. In the first case, delta(s) has a zero at the point given by Eq. Al, so that the equilibrium depends on the initial value of s: if to the left, $\hat{s}=0$; if to the right, $\hat{s}=1$. This solution implies the possibility of a stable minority choice-but for reasons discussed in the text this is not always a realizable long-term outcome. In the final case, the prediction is for stable exclusive choice of the majority alternative.

Bush-Mosteller: stability analysis The analysis for the two-armed-bandit situation can be done in the same way as for ratio invariance. The outcome table is as follows, where $A$ and $B$ are constants, $0<A, B<1$ :

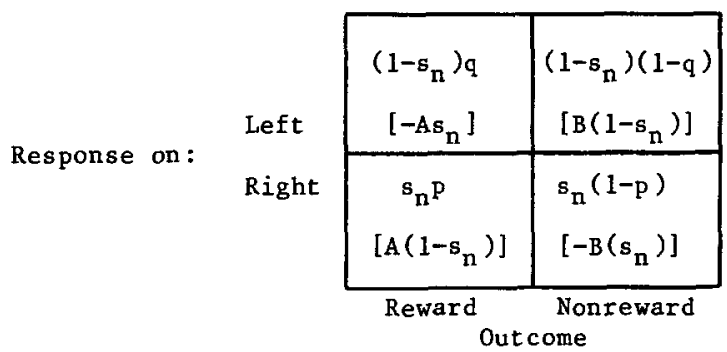


Replacing $s_{n}$ by $s$ for ease of reading, as before, and setting $p=q$, yields the following expectation function

$$
\operatorname{delta}(s)=\operatorname{Aps}(1-s)-B s^{2}(1-p)-\operatorname{Aps}(1-s)+(1-s)^{2}(1-p) B
$$

which simplifies at once to

$$
\operatorname{delta}(s)=B(1-p)(1-2 s) \text {. }
$$

For an equilibrium, delta(s)=0, which yields $s=1 / 2$ as a stable solution for all $p$ values, since the quantity $B(1-p)$ is always $>0$.

The solution for the symmetrical interdependent (SI) schedule in Experiment 1 is the same, indifference, since we merely replace $p$ in Eq. A2 with $p(s)$.

\section{APPENDIX B \\ Interdependent Probabilistic Schedules: Ratio-Invariance Predictions}

In an interdependent schedule the payoff probability for each choice, $p(s)$ or $q(s)$, depends upon choice proportion, s. The equilibrium properties of such a $8 c h e d u l e$ can be derived from Eq. 3 in the text by substituting $p(s)$ and $q(s)$ for $p$ and $q$, and proceeding as before:

$$
\operatorname{de} 1 \mathrm{ta}(s)=s(p(s)+q(s)-2 w)+w-q(s) .
$$

Symmetrical interdependent schedule. For the schedule in Fxperiment $1, p(s)=$ $q(s)$ for all values of $\mathrm{s}$, whence Eq. Bl becomes

$$
\operatorname{delta}(s)=2 s(p(s)-w)+w-p(s) \text {, }
$$

from which it is apparent that delta(s) is always zero when $p(s)=w$, so that if $p(s)=w$ is a possible value for $p(s)$ it is a potential equilibrium.

To find the actual s value corresponding to $p(s)=w$, we need to substitute the actual function to be used for $p(s)$. The left $1 \mathrm{imb}$ of the bilinear function used in Experiment 1 is $p(s)=K s+J$, where $K$ and $J$ are constants and $J$ is small; to an approximation $\mathrm{p}(\mathrm{s})=\mathrm{Ks}$, whence

$$
\operatorname{delta}(s)=2 s(K s-w)+w-K s,
$$

which simplifies to

$$
\operatorname{de} 1 t a(s)=2 K s^{2}-s(2 w+K)+w,
$$

setting Eq. $B 3=0$ yields the roots $s=1 / 2$ and $s=w / K$, which are potential equilibria.

The derivative of Eq. B3 is

$$
\mathrm{d} / \mathrm{ds}[\operatorname{delta}(s)]=4 \mathrm{Ks}-2 \mathrm{w}-\mathrm{K} \text {. }
$$

Substituting $s=1 / 2$ or $s=w / K$ yields the condition that the equilibrium at $s=$ $1 / 2$ is stable iff $w>K / 2$, whereas the equilibrium at $w / K$ is stable iff $w<K / 2$. Since our linear function $p(s)=K s$ extends from $s=0$ to $s=1 / 2$, it will obviously intersect the line $p(s)=w$ iff $w<k / 2$, as Figure 3 in the text indicates.

The analysis for the other limb of the bilinear function in Experiment 1 is the same.

Thus, when $w>K / 2, s=1 / 2$ is the only stable equilibrium; otherwise, there will be a stable equilibrium at the point $s=w / K$. These possibilities are all summarized graphically for the bilinear function in Figure 3. 
Asymmetrical interdependent schedule. For the schedule in Experiment 2 , the payoff probability on the left, $q(s)$ was always twice that on the right: $q(s)=$ $2 p(s)$. Substituting this constraint into Eq. BI yields

$$
\operatorname{delta}(s)=s(3 p(s)-2 w)+w-2 p(s) .
$$

Substituting for $p(s)$ the linear function $(p(s)=$ Ks) shown in Figure 6:

$$
\operatorname{delta}(s)=3 K s^{2}-s 2(w+K)+w .
$$

The two roots of this equation represent potential equilibria, whose stability must be assessed in the usual way. The properties of Eq. B6 can be seen most easily when we equate it to zero (to find the roots) and divide through by $K$; if we denote $\mathrm{w} / \mathrm{K}$ by $\mathrm{V}, \mathrm{Eq}$. B6 then becomes

$$
0=3 s^{2}-2 s(V+1)+V .
$$

If $V=I$ (i.e., $w=k$ ), Eq. B 7 has a root at $s=1$, which has a positive slope; the other root is at $s=1 / 3$. For values of $V>1$ ( $w>K$ ), there is no second root in the interval $0<s<1$, and the first root is always in the interval $1 / 3<$ $s<1 / 2$. When $0<V<1(w<K)$, there are always two roots: a stable root in the interval $0<\mathrm{s}<1 / 3$, and an unstable root in the interval $1 / 2<\mathrm{s}<1$. Examples of delta(s) functions with $w / K$ in each of these three ranges are shown in Figure 7 .

Thus, for the asymmetrical interdependent schedule, ratio invariance predicts that the partial-preference mode must always be in the region $0<s<1 / 2$ and that there will only be a second mode, at exclusive minority choice $(s=1)$, when the partial preference mode is at an $s-v a l u e$ less than $1 / 3$. The relative strength of these two modes depends upon unspecified factors, such as the degree of variability in behavior and the actual increment and decrement functions, $a(s)$ and $b(s)$. We may speculate that the size of the right-hand (minority-exclusivechoice) mode will depend upon the value of the unstable root: the closer it is to 1.0, the less likely that a substantial minority-choice mode will develop.

\section{APPRNDIX $C$ \\ Choice on Concurrent Variable-Interval Schedules}

The reward-following model can be adapted to VI choice by substituting the appropriate molar feedback function (MFF) for $p(s)$ and $q(s)$ in $E q .4$. Because the probability of reward on an interval schedule depends upon the rate of responding, some assumption must be made about rates. We assumed that the sum of rates to the two choices, $x+y=J$, is constant. Choice proportion, $s$, is then equal to $\mathrm{x} /(\mathrm{x}+\mathrm{y})$.

Using the MFF for random responding, reward rate is given by

$$
R(s)=A x /(A+x) \text {, }
$$

where $A$ is the scheduled VI rate (i.e., the maximum possible reward rate). Given that reward probability is just $x / R(x)$, it follows that

and

$$
\begin{aligned}
& p(s)=A /(A+x), \\
& q(s)=B /(B+y) .
\end{aligned}
$$

Substituting $J-x$ for $y$ in Eq. C2, and substituting the result, together with Eq. $\mathrm{Cl}$, into Eq. 4 in the text yields the appropriate delta(s) function. We arrived at the properties described in the text simply by inspecting the plotted functions for the appropriate ranges of $A$ and $B$ values. 